\title{
Diffraction of Horizontally Polarized Ultrasonic Plane Waves on a Periodically Corrugated Solid-Liquid Interface for Normal Incidence and Brewster Angle Incidence
}

\author{
Nico F. Declercq, Member, IEEE, Rudy Briers, Joris Degrieck, and Oswald Leroy
}

\begin{abstract}
The theory, and the use at normal incidence, of shear-vertically polarized waves (with polarization vector in the plane containing the incident wave vector and the normal on the interface) using the mode conversion method has been tackled by others. Here we develop the theory for shear-horizontally polarized incident waves (with polarization vector perpendicular to both the normal on the interface and the incoming wave vector). We take into account normal incidence as well as oblique incidence. For normal incidence, we discover the generation of Love waves. If oblique incidence is considered, we discover the existence of a Brewster angle of incidence, comparable with the Brewster angle in optics, in which a diffraction grating can be used as a polarization filter.
\end{abstract}

\section{INTRODUCTION}

$\mathrm{T}$ HE diffraction of sound by a periodically corrugated 1 surface that is traction free, or is the interface between a solid and a liquid, has been a hot topic for many years [1]-[4], and many methods have been developed in order to tackle the diffraction problem. Claeys et al. [5] and Mampaert and Leroy [6] use one method, the mode conversion theory of diffraction. The method describes the diffracted field as a summation of plane waves, traveling in directions determined by the classical grating equation $\checkmark$ and having amplitudes and phases determined by continuity of normal stresses and normal displacements at the interface. They report calculations tackling diffraction of incident plane waves with polarization perpendicular to the corrugations, i.e., the grooves, on the surface. Their results correspond very well with experiments. The present work reports calculations, using the mode conversion principle used in [5] and [6], for incidence from the solid side and for a polarization parallel to the grooves, i.e., horizontally polarized waves. Contrary to Claeys et al. [5] and Mampaert and Leroy [6], who solely consider normal incidence in their calculations, wejalso take a look at other angles of incidence, more specifically at.an angle that we

Manuscript received March 27, 2002; accepted August 27, 2002.

N. F. Declerq and J. Degrieck are with the Department of Mechanical Construction and Production, Soete Laboratory, Ghent University, 9000 Gent, Belgium (e-mail: NicoF.Declercq@rug.ac.be).

R. Briers is with the RENO Department, Katholieke Hogeschool West Vlaanderen, 8820 Torhout, Belgium.

O. Leroy is with the Interdisciplinary Research Center, Katholieke Universiteit Leuven Campus Kortrijk, 8500 Kortrijk, Belgium.

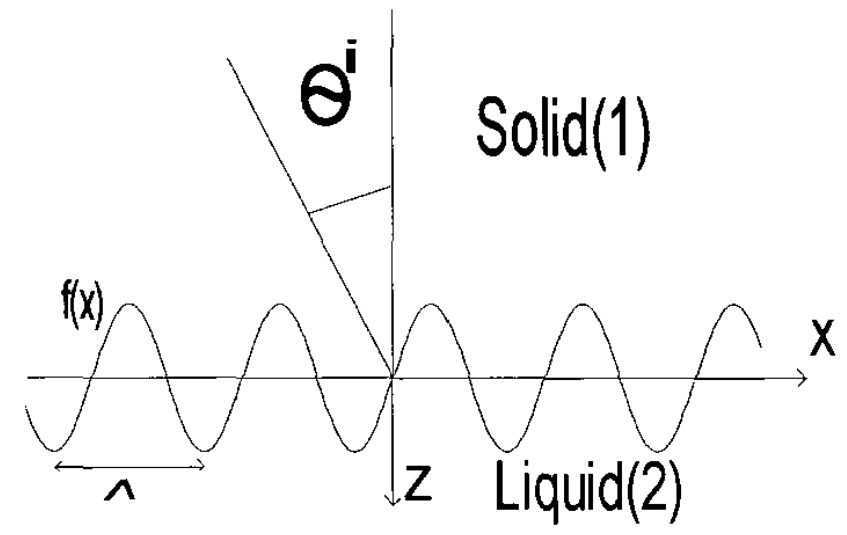

Fig. 1. The geometry of the solid-liquid periodically corrugated interface.

define as the Brewster angle, involving similar effects as in optical scattering at plane interfaces. The geometry of the solid-liquid corrugated interface is depicted in Fig. 1.

\section{There Is No Intersubset Mode Conversion}

Consider a periodically corrugated surface that is the interface between a solid (henceforth upper index 1) and a liquid (henceforth upper index 2), described by:

$$
p(x, z)=f(x)-z=p(x+\Lambda, z)=0,
$$

$\Lambda$ being the period. The wrinkles are directed parallel to the $y$-axis, whence one can easily distinguish between horizontally polarized waves having a polarization vector parallel to the grooves, and vertically polarized waves having a polarization vector perpendicular to the grooves. If $\mathbf{u}^{1}=\mathbf{u}^{i}+\mathbf{u}^{r}$, with $i=$ incident and $r=$ reflected, is the particle displacement in the solid and $\mathbf{u}^{2}=\mathbf{u}^{t}$, with $t=$ transmitted, the particle displacement in the liquid, then we can establish continuity conditions for normal displacements and normal stresses as:

$$
\begin{aligned}
\mathbf{u}^{1} \bullet \operatorname{grad} p & =\mathbf{u}^{2} \bullet \operatorname{grad} p, \\
\sum_{j=1}^{3} T_{k j}^{1} \operatorname{grad}(p)_{j} & =\sum_{j=1}^{3} T_{k j}^{2} \operatorname{grad}(p)_{j},
\end{aligned}
$$


with $\left\lfloor T_{k j}\right\rfloor$ the stress tensor. Relations (2) and (3) yield:

$$
A \mathbf{u}^{i}=B \mathbf{u}^{r}+M \mathbf{u}^{t}
$$

$A, B$, and $M$ being operators depending, due to (1), solely on $\mathrm{x}, \mathrm{z}$ and time. Because $\mathbf{u}^{i}$ also depends exclusively on $\mathrm{x}$, $\mathrm{z}$, and time, relation (4) yields that both $\mathbf{u}^{r}$ and $\mathbf{u}^{t}$ also depend only on $\mathrm{x}, \mathrm{z}$, and time. Taking this into account, we obtain for $(2)$ :

$$
u_{x}^{1} \frac{\partial f}{\partial x}-u_{z}^{1}=u_{x}^{2} \frac{\partial f}{\partial x}-u_{z}^{2}
$$

and for (3), we get:

$$
\begin{aligned}
& {\left[\lambda^{1} \frac{\partial u_{z}^{1}}{\partial z}+\left(2 \mu^{1}+\lambda^{1}\right) \frac{\partial u_{x}^{1}}{\partial x}\right] \frac{\partial f}{\partial x}-\mu^{1}\left[\frac{\partial u_{x}^{1}}{\partial z}+\frac{\partial u_{z}^{1}}{\partial x}\right] } \\
= & {\left[\lambda^{2} \frac{\partial u_{z}^{2}}{\partial z}+\left(2 \mu^{2}+\lambda^{2}\right) \frac{\partial u_{x}^{2}}{\partial x}\right] \frac{\partial f}{\partial x}-\mu^{2}\left[\frac{\partial u_{x}^{2}}{\partial z}+\frac{\partial u_{z}^{2}}{\partial x}\right]_{(6 \mathrm{a})} } \\
& \mu^{1}\left[\frac{\partial u_{y}^{1}}{\partial x} \frac{\partial f}{\partial x}-\frac{\partial u_{y}^{1}}{\partial z}\right]=\mu^{1}\left[\frac{\partial u_{y}^{2}}{\partial x} \frac{\partial f}{\partial x}-\frac{\partial u_{y}^{2}}{\partial z}\right], \quad(6 \mathrm{~b}) \\
& \mu^{1}\left[\frac{\partial u_{z}^{1}}{\partial x}+\frac{\partial u_{x}^{1}}{\partial z}\right] \frac{\partial f}{\partial x}-\left[\lambda^{1} \frac{\partial u_{x}^{1}}{\partial x}+\left(2 \mu^{1}+\lambda^{1}\right) \frac{\partial u_{z}^{1}}{\partial z}\right] \\
= & \mu^{2}\left[\frac{\partial u_{z}^{2}}{\partial x}+\frac{\partial u_{x}^{2}}{\partial z}\right] \frac{\partial f}{\partial x}-\left[\lambda^{2} \frac{\partial u_{x}^{2}}{\partial x}+\left(2 \mu^{2}+\lambda^{2}\right) \frac{\partial u_{z}^{2}}{\partial z}\right]_{(6 \mathrm{c})}
\end{aligned}
$$

The Lamé constants $\lambda$ and $\mu$ are related to the bulk velocities of the respective media (superscript 1 for solid and supcrscript 2 for liquid). Remark that further below, the symbol $\lambda$ also will be used for the wavelength, but never using the superscripts 1 or 2 .

The harmonic nature of the particle vibrations is accomplished by:

$$
\mathbf{u}^{n}=\left[u_{x}^{n}(x, z, t), u_{y}^{n}(x, z, t), u_{z}^{n}(x, z, t)\right] ; \dot{\mathbf{u}}^{n}=i \omega \mathbf{u}^{n},
$$

for $n=i, r$ or $t$.

Conservation of linear momentum $\mathbf{P}$ when particle vibrations are conveyed yields:

$$
\mathbf{P}^{i}=\mathbf{P}^{r}+\mathbf{P}^{t}
$$

The vibrating particles have a mass $m^{s}$ and frequency $\omega$ in the solid and a mass $m^{l}$ and the same frequency $\omega$ in the liquid, whence (8) imparts:

$$
u_{j}^{r}=u_{j}^{i}-\frac{m^{l}}{m^{s}} u_{j}^{t} ; j=x, y, z .
$$

Eq. (6) and (9) yield straightforwardly:

$$
\begin{aligned}
& \mathbf{u}^{i}=\left(0, u_{y}^{i}, 0\right) \Rightarrow\left\{\begin{array}{l}
\mathbf{u}^{t}=(0,0,0), \\
\mathbf{u}^{r}=\left(0, u_{y}^{i}, 0\right)
\end{array},\right. \\
& \mathbf{u}^{i}=\left(u_{x}^{i} 0, u_{z}^{i}\right) \Rightarrow\left\{\begin{array}{l}
\mathbf{u}^{t}=\left(u_{x}^{t}, 0, u_{z}^{t}\right), \\
\mathbf{u}^{r}=\left(u_{x}^{r}, 0, u_{z}^{r}\right)
\end{array},\right.
\end{aligned}
$$

Therefore, it is proved that an incident horizontally polarized wave does not produce any wave in the bulk of the fluid and merely will generate a horizontally polarized reverberated wave; but a vertically polarized wave, either dilatational or vertically shear polarized, will generate a vertical polarized reflected wave and a compressional transmitted wave. Hence, there is no mode conversion from horizontally incident waves to vertical refracted waves and there is also no mode conversion from vertically incident waves to horizontal refracted waves, i.e., there is no mode conversion from the horizontally polarized subset to the vertically polarized subset and vice versa.

\section{Horizontally Polarized Incident Plane WAVES}

In Section II, it is shown that only shear horizontally polarized reflected waves are generated when horizontally polarized (shear) incident waves are considered. The velocity potential for the incoming plane wave is:

$$
\begin{aligned}
& \psi^{i}=C \frac{-i k_{z}^{i}}{\left(k_{x}^{i}\right)^{2}+\left(k_{z}^{i}\right)^{2}} e^{i\left(x k_{x}^{i}+z k_{z}^{i}\right)} \mathbf{e}_{x} \\
& +C \frac{i k_{x}^{i}}{\left(k_{x}^{i}\right)^{2}+\left(k_{z}^{i}\right)^{2}} e^{i\left(x k_{x}^{i}+z k_{z}^{i}\right)} \mathbf{e}_{z}
\end{aligned}
$$

while the velocity potential for the reflected sound is:

$$
\psi^{s}=\xi(x, z) \mathbf{e}_{x}+\zeta(x, z) \mathbf{e}_{z},
$$

where $\mathrm{i}=$ incident horizontally shear polarized, $\mathrm{s}=\mathrm{re}-$ flected horizontally shear, and $\mathrm{C}$ is an amplitude. We decompose the reflected sound field into a Fourier series; therefore, only considering sound that is traveling away from the surface and neglecting sound that is propagating toward the surface due to secondary scattering effects. According to Meecham [7], $\xi(x, z)$ as well as $\zeta(x, z)$ are allowed to be decomposed in a Fourier series [8] whenever the following conditions hold:

$$
\max |f(x)|<\lambda^{i}
$$

where

$$
\lambda^{i} \approx \Lambda
$$

The relations (13) and (14) are called the Lippmann conditions [9] and state that the height of the roughness must be small compared to the wavelength of the ultrasonic wave and the wavelength must be comparable with the roughness period.

Therefore:

$$
\begin{gathered}
\psi^{s}=C \sum_{m=-\infty}^{+\infty} R_{m}\left[\frac{-i k_{z, m}^{s}}{k_{x, m}^{2}+\left(k_{z, m}^{s}\right)^{2}} e^{i\left(x k_{x, m}+z k_{z, m}^{*}\right)} \mathbf{e}_{x}\right. \\
\left.+\frac{i k_{x, m}}{k_{x, m}^{2}+\left(k_{z, m}^{s}\right)^{2}} e^{i\left(x k_{x, m}+z k_{z, m}^{s}\right)} \mathbf{e}_{z}\right]
\end{gathered}
$$


where $m$ is the order of the plane wave functions, $R_{m}$ is the reflection coefficient, $k_{z, m}^{s}$ is the wave number along the $\mathrm{z}$-axis, and $k_{x, m}$ is the wave number along the x-axis, of the $m^{\text {th }}$ order plane wave. $k_{x, m}$ and $k_{z, m}^{s}$ are related to each other through the wave speed. The classical grating equation holds:

$$
\sin \theta_{m}^{s}=\sin \theta^{i}+m \frac{\lambda^{s}}{\Lambda} \Leftrightarrow k_{x, m}=k_{x}^{i}+m \frac{2 \pi}{\Lambda},
$$

where $\theta^{i}$ is the incidence angle with the z-axis, $\theta_{m}^{s}$ is the reflection angle with the $\mathrm{z}$-axis of the $m^{\text {th }}$ order reflected plane wave, and $\lambda^{s}$ is the wavelength of the diffracted wave, governed by the frequency (not to be confused with the Lamé constant $\lambda^{1}$ or $\lambda^{2}$ ). Because omitting the time dependence,

$$
\mathbf{u}^{1}=C\left\lfloor e^{i\left(x k_{x}^{i}+z k_{z}^{i}\right)}+\sum R_{m} e^{i\left(x k_{x, m}+z k_{z, m}^{s}\right)}\right\rfloor \mathbf{e}_{y}
$$

The continuity conditions (2) and (3), at $z=f(x)$, demand:

$$
T_{y x}^{1} \operatorname{grad}(p)_{x}+T_{y z}^{1} \operatorname{grad}(p)_{z}=0
$$

or likewise,

$$
\begin{aligned}
& {\left[\frac{\partial f}{\partial x} k_{x}^{i}-k_{z}^{i}\right] e^{i\left[x k_{x}^{i}+f(x) k_{z}^{i}\right]}} \\
& =-\sum\left[\frac{\partial f}{\partial x} k_{x, m}-k_{z, m}^{s}\right] R_{m} e^{i\left[x k_{x, m}+f(x) k_{z, m}^{s}\right]}
\end{aligned}
$$

Due to the periodicity of the tracks on the surface, both sides of (19) are periodic, whence we can write them as a Fourier series in $e^{i k_{x, n} x}$ with $k_{x, n}$ defined in (16) for ' $\mathrm{m}$ ' replaced by ' $n$ '. Then, (19) holds if, and only if, the Fourier coefficients of both sides in (19) are equal to each other. This results in:

$$
\left(k_{x}^{i} k_{x, n}-\frac{\omega^{2}}{v_{s}^{2}}\right) I_{i, n}^{k_{z}^{i}}=\sum R_{\tau n}\left[-k_{x, m} k_{x, n}+\frac{\omega^{2}}{v_{s}^{2}}\right] \underset{m, n}{I_{(20}^{s}}
$$

where

$$
\begin{aligned}
I_{i, n}^{k_{z}^{i}} & =\frac{1}{k_{z}^{i}} \int_{0}^{\Lambda} e^{i\left[\left(k_{x}^{i}-k_{x, n}\right) x+f(x) k_{z}^{i}\right]} d x, \\
I_{m, n}^{s} & =\frac{1}{k_{z, m}^{s}} \int_{0}^{\Lambda} e^{i\left[\left(k_{x, m}-k_{x, n}\right) x+f(x) k_{z, m}^{s}\right]} d x,
\end{aligned}
$$

involving:

$$
k_{x, m}^{2}=\frac{\omega^{2}}{\left(v^{s}\right)^{2}}-\left(k_{z, m}^{s}\right)^{2}
$$

and

$$
\left(k_{x}^{i}\right)^{2}=\frac{\omega^{2}}{\left(v^{s}\right)^{2}}-\left(k_{z}^{i}\right)^{2}
$$

in which $v^{s}$ is the shear wave velocity. As a consequence of the disability of fluids to carry shear waves, it is seen from (20) that the theory also is valid for pressure release surfaces. Eq. (20) is valid for each $m$ and $n$ and, therefore, generates a transformation, having an infinite number of coefficients, of an infinite number of variables $R_{m}$. The discrete nature of this transformation enables us to calculate the value of the variables $R_{m}$ using a computer procedure, whence it also is necessary to truncate the set of $m$ and $n$ values. The infinite transformation may be reduced to a finite transformation due to energy considerations. That is because it is physically necessary that only a finite number of $R_{m}$ are significant as the incoming sound solely delivers finite energy per unit of time. We have performed calculations for $\mathrm{m}=1,2,3, \ldots$, and we have noticed that the alteration of the results when $m$ or $n$ exceed 3 does not exceed the smallest representable number of our computer. Therefore, it can be concluded that the results have converged as perfectly as possible if we chop $\left\{R_{m}\right\}$ at $|\mathrm{m}|=3$. For practical reasons, however, we have chopped $\left\{R_{n}\right\}$ at $|\mathrm{m}|=8$. Therefore, we only consider $m, n \in\{-8, \ldots, 8\}$, i.e., a transformation of 17 by 17 is considered and also 17 unknown variables $R_{m}$. Even though the rapid convergence of our results makes numerical error estimation superfluous, it is interesting to remark that such an estimation can be made when necessary through power flow considerations [6] resulting in:

$$
\sum\left|\cos \theta_{m}^{s}\right|\left|R_{m}\right|^{2}=\left|\cos \theta^{i}\right|
$$

for the summation over all $m$ for which $\sin \theta_{m}^{s}$ exists, determined by the classical grating equation (16).

\section{Calculations}

\section{A. Normal Incidence of Horizontally Polarized Plane Waves}

Following Claeys et al. [5] and Mampaert and Leroy [6] who found anomalies in reflection spectra for vertically polarized waves that correspond to generated surface waves, we have calculated reflection spectra for horizontally polarized waves. Fig. 2 shows that, for a horizontally polarized plane wave with $0 \mathrm{~dB}$ intensity, striking a sine shaped interface between stainless steel and water with $\max |f(x)|=30 \mu \mathrm{m}$ and $\Lambda=350 \mu \mathrm{m}$, that at $8.83 \mathrm{MHz}$, the zero order shows an anomaly accompanied by a maximum for the first order wave. The maximum exceeds $0 \mathrm{~dB}$, whence it must be an inhomogeneous plane wave in agreement with the fact that, up to and beyond $8.83 \mathrm{MHz},(23)$ shows that $k_{z, 1}^{s}$ is pure imaginary. Because this surface wave is pure shear polarized, it is a Love-like wave. Fig. 3 shows that, at $8.83 \mathrm{MHz}$ the phase of the different orders undergo a strong phase shift. For low frequencies there is no diffraction because waves having a large wavelength are not susceptible for small corrugations, and hence all the energy (see Fig. 2) stays in the zero order wave during 


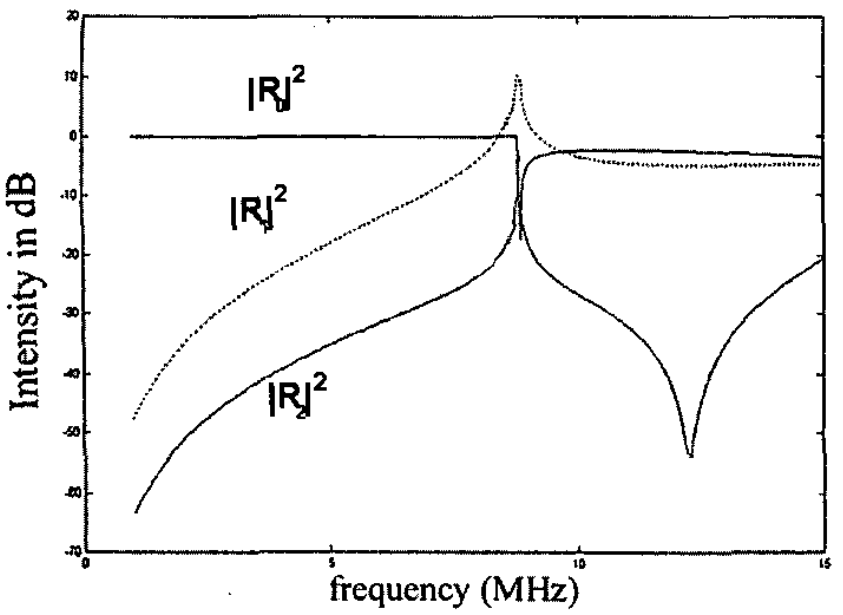

Fig. 2. The intensities of the three first reflected orders in decibels.

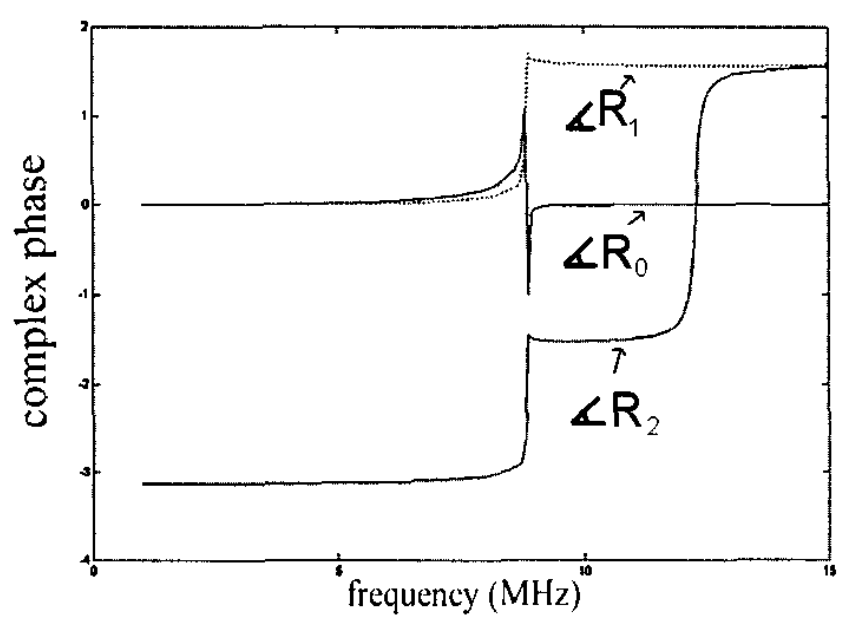

Fig. 3. The phase of the three first reflected orders.

reflection. The reflected wave shows no phase difference with the zero phase of the incident wave. For higher frequencies, the incoming energy is distributed in the excited orders, and the specular zero order reflected wave shows a phase difference with the incoming wave.

\section{B. Incidence at Brewster Angle of Shear Polarized Plane Waves}

In optics, it is well-known [10] that, for arbitrarily shear polarized light, incident at the Brewster angle, the specular reflected light has a polarization parallel to the interface and perpendicular to the incident light ray. In acoustics, we can, for a periodically corrugated surface, also define a Brewster angle $\theta^{i}$ in solids as the angle of the incident sound, at a chosen frequency and with arbitrary shear polarization, for which the zero order reflected sound, in the $-\theta^{i}$ direction, is horizontally polarized. In other words, the zero order reflected sound will contain a considerable amplitude $R_{0}$ of a horizontally polarized reflected wave and a negligible amplitude $S_{0}$ of a vertically polarized reflected shear wave. There also will be a longitudinal polarized wave, but it will propagate in a different direction and can be filtered out if bounded beams are used instead of plane waves. We know [6] that, for normal incidence, vertically polarized incident waves will generate ScholteStoneley waves (SST) at certain frequencies freq $_{\mathrm{SST}, \mathrm{m}}, \mathrm{m}$ being the diffraction order that absorb much, if not all, of the incident vertically polarized sound. We also know from the classical grating equation (16) that in case SST are generated, the classical grating equation for an incident plane wave with $k_{x}^{i}$ different from zero may be written as:

$$
k_{x, m}^{\mathrm{SST}}=k_{x}^{i}+m \frac{2 \pi}{\Lambda} .
$$

As mentioned in Section IV-A, SST contain an imaginary z-component and a real $\mathrm{x}$-component of their wave vector. Therefore, the wave vector is complex. From the dispersion equation in the complex waves theory, which can be found in numerous papers concerning such waves, we know that we can write any wave vector as the angular frequency divided by a complex wave velocity. Therefore, we may express the real component of the wave vector (i.e., $k_{x, m}^{\mathrm{SST}}$ ) as the angular frequency divided by its real (i.e., measurable) velocity $v$. We must, however, also take into account the direction of propagation (plus for positive $\mathrm{m}$, minus for negative $\mathrm{m}$ ). If we also assume that the velocity $v$ of SST is independent of the frequency (i.e., $v$ is equal to a frequency independent value $v^{\mathrm{SST}}$ ) then:

$$
k_{x, m}^{\mathrm{SST}}=\operatorname{sign}(m) \frac{2 \pi \mathrm{freq}}{v^{\mathrm{SST}}} .
$$

while for the (real) incident plane waves:

$$
k_{x}^{i}=\frac{2 \pi \text { freq }}{v^{s}} \sin \theta^{i},
$$

hence relations (26)-(28) result in:

$$
\text { freq }=\frac{m}{\Lambda} \frac{v^{\mathrm{SST}} v^{s}}{\operatorname{sign}(m) v^{s}-v^{\mathrm{SST}} \sin \theta^{i}},
$$

and

$$
\theta^{i}=\operatorname{sign}(m) \arcsin \left[\left(\frac{\text { freq }}{v^{\text {SST }}}-\frac{|m|}{\Lambda}\right) \frac{v^{s}}{\text { freq }}\right]
$$

Relations (29) and (30) tell us at what angle $\theta^{i}$ the surface must be insonified with sound of frequency freq if SST are to be generated, and vice versa. It is this $\theta^{i}$ that we define as the Brewster angle of incidence, because zero order reflected sound will be created that, due to the extremely small amplitude of the reflected vertically polarized waves, does not, or almost not, contain any vertically polarized waves, and thus solely horizontally polarized waves.

We now will see some consequences of the so far found formulas (29) and (30). It is important to notice that, for normal incidence [6], an SST generating frequency generates both an m'th order and a $-\mathrm{m}^{\prime}$ th order SST. For oblique incidence, however, as seen from (29) and (30) for 
a certain angle of incidence, the $m$ 'th order and the $-m^{\prime}$ th order will be generated at different frequencies and for a chosen frequency, a $\theta^{i}$ will produce the $\mathrm{m}^{\prime}$ th order and $-\theta^{i}$ will generate the $-m^{\prime}$ th order. It is equally important to remark that only a $\theta^{i}$ can be found for a given frequency freq, using (30) if:

$$
\begin{aligned}
0<\left(\frac{v^{s}}{v^{s}+v^{\mathrm{SST}}}\right) \text { freq }^{\mathrm{SST}} & \leq \text { freq } \\
& \leq \text { freq }^{\mathrm{SST}}\left(\frac{v^{s}}{v^{s}-v^{\mathrm{SST}}}\right),
\end{aligned}
$$

freq ${ }^{\mathrm{SST}}$ being the frequency at which SST of the considered $m$ 'th order are generated using normal incidence and that for a given angle of incidence $\theta^{i}$, a positive frequency can only be found, using (29) if:

$$
\sin \theta^{i} \operatorname{sign}(m)<\frac{v^{s}}{v^{\mathrm{SST}}}
$$

It also is seen from (30) that:

$$
\begin{aligned}
& \operatorname{sign}\left(\theta^{i}\right)=\operatorname{sign}(m) \Leftrightarrow \text { freq } \geq \text { freq }^{\mathrm{SST}}, \\
& \operatorname{sign}\left(\theta^{i}\right)=-\operatorname{sign}(m) \Leftrightarrow \text { freq } \leq \text { freq }^{\mathrm{SST}} .
\end{aligned}
$$

One consequence of (31) is the necessity of $v^{\mathrm{SST}}<v^{s}$, by which (32) is fulfilled too. Calculations have convinced us that the defined Brewster angle (30) never generates horizontally polarized surface waves because the Love wave frequency is always different from the SST frequency, whence $\theta^{i}$ is clearly a Brewster angle in every sense. An example is given in Fig. 3 for a shear polarized wave, of frequency $7 \mathrm{Mhz}$, isonifying a sine-shaped stainless steelwater interface with $\Lambda=350 \mu \mathrm{m}$ and $\max |f(x)|=30 \mu \mathrm{m}$, for which (31) gives:

$$
5.72 \mathrm{MHz} \leq \text { freq } \leq 16.16 \mathrm{MHz},
$$

$v_{2}^{\mathrm{SST}}=1478.87 \mathrm{~m} / \mathrm{s}$ was used, obtained from calculations using normal incidence at the second order SST generating frequency of $8.45 \mathrm{MHz}$. Formula (30) for $7 \mathrm{MHz}$ predict $\theta^{i}=26^{\circ}$, which is indeed part of the calculated broad valley of almost zero amplitude as shown in Fig. 4, but the exact minima (i.e., the deepest spot in that broad valley) of $\left|S_{0}\right|^{2}$ for $7 \mathrm{MHz}$, is at $\theta^{i}=29.5^{\circ}$, which would have been predicted by formula $(30)$ if $v_{2}^{\mathrm{SST}}=1523.80 \mathrm{~m} / \mathrm{s}$ was used. Because normal spectra predict $v_{2}^{\mathrm{SST}} \approx v_{1}^{\mathrm{SST}}$, we do not believe that the SST velocity alters because of a slightly different frequency, but due to a different angle of incidence. It is as if the continuously and obliquely insonifying sound field stimulates the SST to travel faster than in the event of normal incidence.

\section{CONCLUSIONS}

A method has been developed to tackle the diffraction of horizontally polarized shear incident plane waves at a periodically corrugated interface between a solid and an ideal

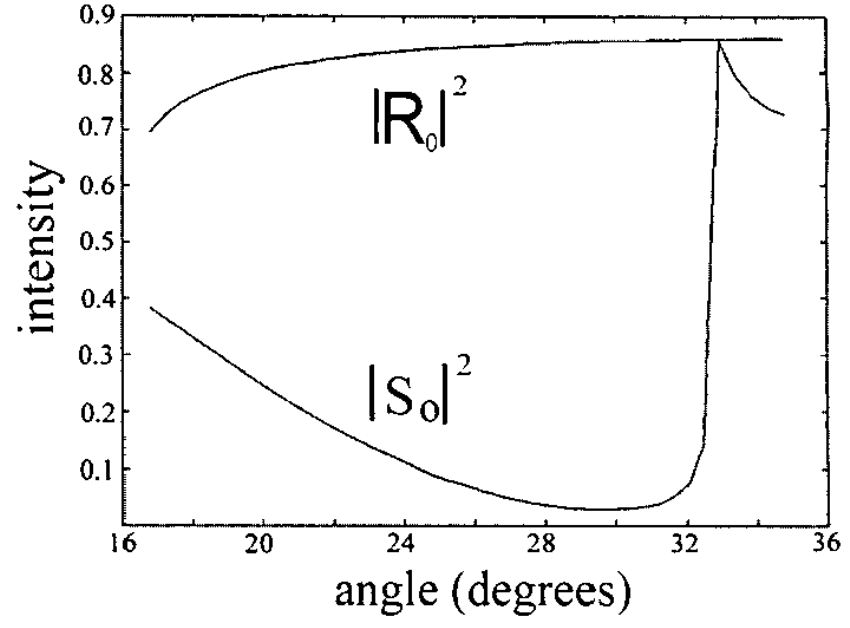

Fig. 4. The intensity of $S_{0}$ and $R_{0}$ as a function of the angle of incidence. $S_{0}$ shows a minimum at $29.5^{\circ}$, which we define as the Brewster angle.

liquid. The creation of Love-like waves has been predicted. The existence of a Brewster angle has been discovered, and calculations using vertically polarized incident waves with continuity conditions obtained by Mampaert and Leroy [6] show indications that Scholte-Stoneley waves generated by diffraction have larger velocities for oblique incidence than for normal incidence.

\section{ACKNOWLEDGMENTS}

The authors wish to thank The Flemish Institute for the Encouragement of the Scientific and Technological Research in Industry (I.W.T.) for sponsoring and the Ghent University for its hospitality. The authors also wish to express their gratitude to the reviewers for their constructive comments.

\section{REFERENCES}

[1] R. Briers, O. Leroy, O. Poncelet, and M. Deschamps, "Experimental verification of the calculated diffraction field generated by inhomogeneous waves obliquely incident on a periodically rough liquid-solid boundary," J. Acoust. Soc. Amer., vol. 106, no. 2, pp. 682-687, 1999.

[2] J. A. DeSanto and P. A. Martin, "On angular-spectrum representations for scattering by infinite rough surfaces," Wave Motion, vol. 24, pp. 421-433, 1996.

[3] R. C. McNamara and J. A. DeSanto, "Numerical determination of scattered field amplitudes for rough surfaces," J. Acoust. Soc. Amer., vol. 100, no. 6, pp. 3519-3526, 1996.

[4] P. E. McSharry, D. T. Moroney, and P. J. Cullen, "Wave scattering by a two-dimensional pressure-release surface based on a perturbation of the Green's function," J. Acoust. Soc. Amer., vol. 98 , no. 3, pp. 1699-1716, 1995.

[5] J. M. Claeys, O. Leroy, A. Jungman, and L. Adler, "Diffraction of ultrasonic waves from periodically rough liquid-solid surface," J. Appl. Phys., vol. 54, no. 10, pp. 5657-5662, 1983.

[6] K. Mampaert and O. Leroy, "Refiection and transmission of normally incident ultrasonic waves on periodic solid-liquid interfaces," J. Acoust. Soc. Amer., vol. 83, no. 4, pp. 1390-1398, 1988 . 
TABLE I

Calculated Frequencies and Velocities $\left(v_{L}\right)$ of Love Waves for Different Solid-Water Interfaces with max $|f(x)|=35 \mu \mathrm{m}$ AND $\Lambda=350 \mu \mathrm{m}$.

\begin{tabular}{lccccc}
\hline & $\rho\left(\mathrm{kg} / \mathrm{m}^{3}\right)$ & $v_{d}(\mathrm{~m} / \mathrm{s})$ & $v_{s}(\mathrm{~m} / \mathrm{s})$ & Love-frequency $(\mathrm{MHz})$ & $v_{L}(\mathrm{~m} / \mathrm{s})$ \\
\hline Stainless steel & 7850 & 5700 & 3100 & 8.83 & 3094.32 \\
Brass & 8100 & 4700 & 2100 & 5.98 & 2092.49 \\
Perspex & 1060 & 2350 & 1150 & 3.27 & 1144.13 \\
\hline
\end{tabular}

[7] W. C. Meecham, "Variational method for the calculation of the distribution of energy reflected from a periodic surface," J. Appl. Phys., vol. 27, pp. 361-367, 1956.

[8] J. L. Uretsky, "The scattering of plane waves from periodic surfaces," Ann. Phys., vol. 33, pp. 400-427, 1965.

[9] B. A. Lippmann, "Note on the theory of gratings," J. Opt. Soc. Amer., vol. 43, p. 408, 1953.

[10] D. Halliday, R. Resnick, and J. Walker, Fundamentals of Physics. 4th ed d. 1993, pp. 1017-1018.

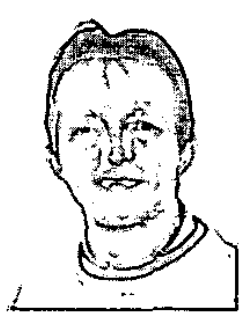

Nico F. Declercq (M'01) was born in Kortrijk, Belgium, in 1975 and has B.Sc. and M.Sc. degrees in astrophysics from the Catholic University of Leuven, Belgium

He began working as a Ph.D. student in the Physical Acoustics and Acousto-Optics Research Group at the Interdisciplinary Research Center at the Catholic University of Leuven Campus Kortrijk, Kortrijk, Belgium and moved to the Ghent University in October 2001. He continues his research with fnancial support of the Flemish Institute for the Encouragement of the Scientific and Technological Research in Industry (I.W.T.). He is a member of the Institute of Electric and Electronic Engineering, the Acoustical Society of America (ASA), the Belgian Acoustical Society (ABAV), the Société Française d'Acoustic (SFA), and the Russian Acoustical Society (RAS). He has won the Best Presentation Award at the 2nd FTW Ph.D. symposium (Ghent University 2001)

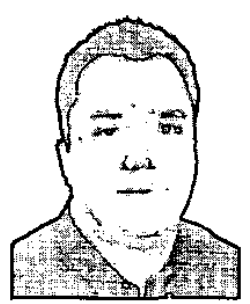

Rudy Briers was born in Geel, Belgium, on October 9, 1965. He has B.Sc. and M.Sc. degrees in mathematics, is aggregated for teaching in higher education, and has a Ph.D. in science (acoustics) from the Catholic University of Leuven, Belgium. He has been an assistant professor in the Department of Astronorny and the Department of Physical Acoustics. After receiving his Ph.D. degree, he worked as a postdoctoral fellow and was a driving force at the Physical Acoustics and Acousto-Optics Research Group of Prof. Oswald Leroy, with financial support of the Flanders Institute for Scientific Research and Technology (IWT) and the Belgian National Fund for Scientific Research (FWO). At present, he is a lecturer in mathematics and computer sciences at the Katholieke Hogeschool Zuid-West Vlaanderen, Torhout, Belgium (KATHO) and is a guest professor of statistics in medical science at the Catholic University of Leuven (KUL).

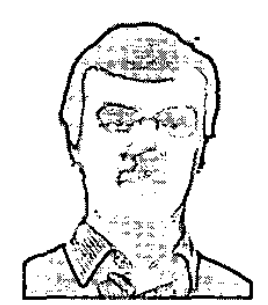

Joris Degrieck was born in Veurne, Belgium, on February 10, 1959. He has an M.Sc. degree in mechanical engineering and a Ph.D. degree in applied science (mechanical engineering) from the Ghent University, Ghent, Belgium.

From 1986 until 1991 he was assistant and lecturer at the Ghent University. From 1991 until 1996, he was a research engineer and project leader at the Research Institute of the Belgian Metalworking Industries, Ghent, Belgium (WTCM-CRIF). From 1993 until 1996, he was, respectively, part-time professor, teaching Mechanics of Fibre Reinforced Materials, and professor, teaching Composite Materials and Kinematics and Dynamics of Mechanisms, at the Ghent University. His main research activities are situated in damage models for composites under dynamic pressure, nondestructive monitoring of deformations in composite structural elements by means of embedded optical fiber sensors, and nondestructive characterization of the elastic properties of composite laminates by means of ultrasound.

He has won the Vreedenburgh Prize (TU Delft, The Netherlands, 1991) and the prize of the Belgian Rayal Academy for Arts, Letters and Sciences-Class Sciences (1991).. He is a member of the Flemish Engineering Association (KVIV), the Society for the Advancement of Material and Process Engineering, the Materials and Processes Society (SAMPE), the Belgian Research Centre for Pipes and Equipment Becetel, the Belgian Society of Mechanical and Environmental Engineering (BSMEE), the European Society for Composite Materials (ESCM), and he is an affiliate member of the Institute of Materials.

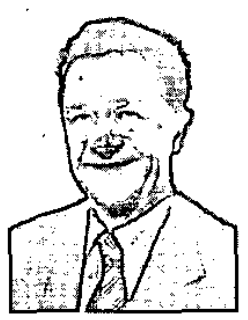

Oswald Leroy was born in Passendale, Belgium, in 1936. He received his Ph.D. degree in mathematical physics from the Ghent University. His main fields of interest are acoustooptics and physical acoustics.

He worked as an assistant at the Ghent University from 1966 to 1972 and has been a professor at the Catholic University of Leuven since 1972, where he established and directed the Physical Acoustics and Acousto-Optics Research Group at the Interdisciplinary Research Center. He was a guest professor at Université de Paris 7 and Université de Bordeaux in France, University of Tennessee in the United States, and Tokyo Institute of Technology in Japan. He has becn a referee for many international journals. He received Nato Grants in cooperation with Georgetown University, University of Tennessee, and University of Houston.

He is a member of the Acoustical Society of America, the Institute of Electric and Electronic Engineering, the Acoustical Society of India, Société Française d'Acoustic, and the Belgian Acoustical Society. He received an Honorary Doctorate from the University of Gdansk, Poland, in 1991. He has won the Médaille étrangère of the French Acoustical Society in 2001. He accorded his Emeritus Status in October 2001. 